\title{
Changes to Vascular Supply of Auditory Cortex Following Chronic Conductive Hearing Loss
}

\author{
R.J. Mount, A.T.D. Licup-Bravo, M. Pienkowski, S. Daniel, and R.V. Harrison
}

Auditory Science Laboratory, Brain \& Behaviour Division and Dept. of Otolaryngology - Head and Neck Surgery, Hospital for Sick Children, 555 University Ave., Toronto, Canada M5G 1X8

Normal auditory input is an essential influence on the development and maturation of the central auditory pathways. Thus, after sensorineural hearing loss from an early age, animal model studies show an abnormal neural reorganization of auditory cortex [1,2]. There is also evidence that long term conductive hearing loss in infants, e.g. from chronic or repetitive otitis media, can contribute to language delay and other cognitive impairments. Such conductive loss can significantly reduce sound evoked neural activity patterns in auditory cortex, particularly that contralateral to the hearing loss ear. We have argued elsewhere that the structural development of local blood vessels in cortex is dependant on local metabolic demands, and therefore on neural activity levels [3]. This leads us to our experimental question. If chronic conductive hearing loss results in reduced neural activity levels in cortex, is there a corresponding alteration to the structure of blood capillary beds in auditory areas?

This study explores alterations in blood vascularity of auditory cortex in a model of unilateral chronic conductive hearing loss. In newborn chinchilla pups, unilateral conductive hearing loss was induced by plugging the left external auditory meatus at birth, followed by a left ear ossiculectomy at 1 to 2 weeks of age. Right ears were used as a within animal control. At one year post surgery we use electrophysiological methods to assess auditory thresholds and to accurately locate primary auditory areas of temporal cortex. Vasculature corrosion casts of the brain are prepared and surface vasculature aligned with that recorded during electrophysiologic mapping to identify primary auditory cortex (AI) and surrounding nonAI regions of the cast [3]. Figure 1 shows the capillary structure of AI and nonAI regions from both control and deprived temporal cortex. Stereopair scanning electron photomicrographs were taken from each area and analyzed for the following parameters of vascularity: capillary diameter, capillary density (segments/area), number of branching points per area, and diffusion distance (1/sq.rt \# segments). In normal hearing animals differences in vascular parameters between left and right cortices are found but the ratio of the parameters between AI and nonAI remain constant [unpublished findings].

Analysis results are given in Table 1. In the control cortex the microvasculature of AI could be differentiated from nonAI on the basis of significantly greater capillary diameters and capillary density (segments/area). The diffusion distance was significantly less in AI than in nonAI. In the deprived cortex there were no significant differences between the AI and nonAI regions. Branching patterns were not significantly different in either case.

There are normally significant differences in capillary bed structure between AI cortex and surrounding areas, as a result of the higher levels of neural activity in AI compared with nonAI regions. On the cortical side receiving reduced auditory input because of conductive hearing loss, these capillary bed differences between AI and surrounding areas are reduced resulting in an AI region which closely resembles the nonAI. 
References

[1] R.V. Harrison et al., Hear Res. 54 (199) 11.

[2] S. Stanton and R.V. Harrison, J Comp Neurol. 426 (2000) 117.

[3] R.V. Harrison et al., Cereb Cortex. 12 (2002) 225.
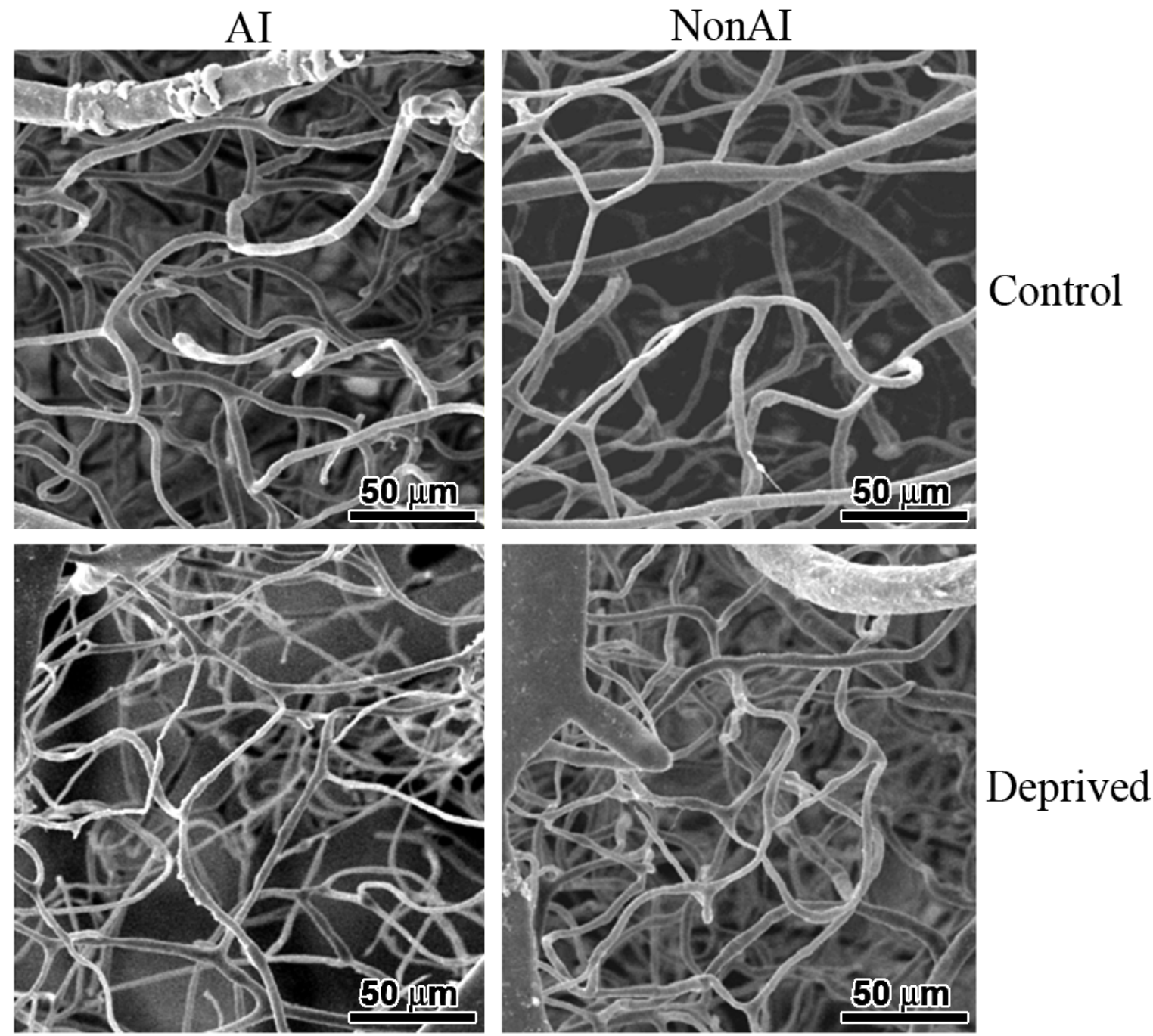

Fig. 1. Representative capillary casts of AI and nonAI regions from control and deprived cortex.

TABLE 1. Comparison AI and nonAI regions of auditory cortex from control and auditory deprived cortices $(*$ significant difference).

\begin{tabular}{lrrrrrrrr}
\hline Parameter & \multicolumn{3}{c}{ Control cortex } & \multicolumn{4}{c}{ Deprived cortex } \\
& AI & nonAI & Ratio & T test & AI & nonAI & Ratio & T test \\
\hline Capillary diameter $(\mu \mathrm{m})$ & 3.98 & 3.58 & 1.112 & $<0.0001^{*}$ & 3.70 & 3.63 & 1.019 & 0.1217 \\
Segments/0.1 sq mm & 196 & 171 & 1.147 & $0.0045^{*}$ & 214 & 203 & 1.053 & 0.5218 \\
Diffusion distance $(\mu \mathrm{m})$ & 7.17 & 7.68 & 0.934 & $0.0057^{*}$ & 7.04 & 7.18 & 0.981 & 0.6417 \\
Branching points $/ 0.1 \mathrm{sq} \mathrm{mm}$ & 88.67 & 78.79 & 1.125 & 0.0848 & 94.31 & 89.48 & 1.054 & 0.5284 \\
\hline
\end{tabular}

